\title{
Nocturnal thoracoabdominal asynchrony in house dust mite-sensitive nonhuman primates
}

This article was published in the following Dove Press journal:

Journal of Asthma and Allergy

27 July 2010

Number of times this article has been viewed

\section{Xiaojia Wang \\ Shaun Reece \\ Stephen Olmstead \\ Robert L Wardle \\ Michael R Van Scott \\ Department of Physiology, East \\ Carolina University, Greenville, North \\ Carolina, USA}

Correspondence: Michael R Van Scott Department of Physiology, The Brody School of Medicine, East Carolina University, 6N98 Brody Building, Greenville, NC 27834, USA

Tel + I 2527443654

Fax +I 2527443460

Email vanscottmi@ ecu.edu

\begin{abstract}
Nocturnal bronchoconstriction is a common symptom of asthma in humans, but is poorly documented in animal models. Thoracoabdominal asynchrony (TAA) is a noninvasive clinical indication of airway obstruction. In this study, respiratory inductive plethysmography (RIP) was used to document nocturnal TAA in house dust mite (HDM)-sensitive Cynomolgus macaques. Dynamic compliance $\left(\mathrm{C}_{\mathrm{dyn}}\right)$ and lung resistance $\left(\mathrm{R}_{\mathrm{L}}\right)$ measured in anesthetized animals at rest and following exposure to HDM allergen, methacholine, and albuterol were highly correlated with three RIP parameters associated with TAA, ie, phase angle of the rib cage and abdomen waveforms (PhAng), baseline effort phase relation (eBPRL) and effort phase relation (ePhRL). Twenty-one allergic subjects were challenged with HDM early in the morning, and eBPRL and $\mathrm{PhRL}$ were monitored for 20 hours after provocation. Fifteen of the allergic subjects exhibited gradual increases in eBPRL and ePhRL between midnight and $6 \mathrm{am}$, with peak activity at $4 \mathrm{am}$. However, as in humans, this nocturnal response was highly variable both between subjects and within subjects over time. The results document that TAA in this nonhuman primate model of asthma is highly correlated with $\mathrm{C}_{\mathrm{dyn}}$ and $\mathrm{R}_{\mathrm{L}}$, and demonstrate that animals exhibiting acute responses to allergen exposure during the day also exhibit nocturnal TAA.
\end{abstract}

Keywords: nocturnal asthma, late phase asthmatic response, respiratory inductive plethysmography

\section{Introduction}

Allergic asthma is a common disease in developed countries, with an incidence of approximately $8 \%$ in the US. ${ }^{1}$ Nocturnal symptoms of asthma are common and include bronchoconstriction, airway inflammation and hyperreactivity, dyspnea, cough, and apnea. ${ }^{2-6}$ The incidence and magnitude of nocturnal asthma is variable. Approximately $70 \%$ of asthmatics experience nocturnal symptoms at least once per week, and 10\% experience nocturnal symptoms at least three times per week. ${ }^{7}$ Nocturnal symptoms can be severe, and a high incidence of respiratory arrest and deaths due to asthma is seen between midnight and 8 am. ${ }^{8-10}$ The mechanisms underlying nocturnal asthma have not been elucidated.

Early and late asthmatic responses have been routinely investigated using animal models, but nocturnal symptoms are poorly documented in these models due in part to the nocturnal nature of rodents and to the techniques commonly used to assess bronchoconstriction in larger diurnal laboratory animals. Respiratory inductive plethysmography (RIP) is a noninvasive technique for monitoring breathing patterns ${ }^{11-26}$ and may be applicable to nocturnal studies in large animals. RIP uses deflection of the ribcage and abdomen to estimate changes in lung volume and airflow, and the 
phase relationship of abdominal and thoracic movements. Restricting airflow through the airways causes movements of the thorax and abdomen to be out of phase, giving rise to thoracoabdominal asynchrony (TAA). TAA is a common clinical indicator of airway obstruction, ${ }^{15,27-31}$ and in this study was used to detect nocturnal respiratory disturbances.

Nonhuman primates (NHP) exhibit a high level of similarity to humans in genetic, anatomic, and physiologic aspects, and are well suited to modeling human diseases (eg, asthma). ${ }^{32}$ The house dust mite (HDM) model of allergic asthma in Cynomolgus macaques reproduces the key features of human asthma, including elevated serum IgE levels, $\beta$-agonist reversible bronchoconstriction, and latephase eosinophilic inflammation. ${ }^{33}$ In addition, the airways become hyperresponsive to nonspecific bronchoconstrictors including histamine, methacholine, and adenosine. ${ }^{33,34} \mathrm{CD}^{+}$ $\mathrm{T}$ lymphocytes and natural killer $\mathrm{T}$ cells in bronchoalveolar lavage fluid are increased, and Th2 lymphocyte cytokines are upregulated. ${ }^{35}$ The severity of the asthma phenotype in the NHP model depends on the schedule of allergen challenges and the provocative dose of HDM. At an exposure periodicity of 4-6 week intervals, as used in this study, the animals have minimal symptoms between exposures, but on the day of provocation, exhibit a severe acute asthmatic response, including decrease in arterial $\mathrm{O}_{2}$ saturation to less than $80 \%$ and in some animals less than $70 \%$. Upon resolution of the acute response, the animals develop eosinophilia that is equivalent to what is observed following segmental allergen challenge in asthmatic humans. ${ }^{36}$ As in humans, the symptoms are attenuated with corticosteroids. ${ }^{33,35}$ Extensive terminal protocols have not been conducted on these animals, but in a few animals that have been examined, airway wall remodeling and mucosal eosinophilia has been observed. ${ }^{33}$ These characteristics are consistent with mild to moderate intermittent asthma in humans that is well controlled by $\beta$-agonists and corticosteroids.

The goal of this study was to determine if aerosol allergen exposure elicits a delayed, nocturnal response in HDMsensitive NHP. Nonallergic and HDM-sensitive animals were identified from within an existing colony of Cynomolgus macaques. Correlation between RIP measurements and direct measures of lung resistance $\left(\mathrm{R}_{\mathrm{L}}\right)$ and dynamic compliance $\left(\mathrm{C}_{\mathrm{dyn}}\right)$ were explored by inducing acute bronchoconstriction with aerosolized methacholine and HDM allergen. RIP parameters indicative of TAA exhibited a high degree of correlation with the conventional measures of airway function. The animals were subsequently challenged with aerosolized saline or HDM allergen, and TAA was monitored by RIP for 20 hours to detect nocturnal disturbances in respiration.

\section{Methods Overview}

Cynomolgus macaques with documented sensitivity to aerosolized HDM for two or more years prior to the study were identified within an existing colony at East Carolina University. Animals with no history of allergic sensitivity were used as nonallergic controls. Allergy status at the time of the study was confirmed by serum levels of HDM-specific IgE, early-phase bronchoconstriction to aerosolized HDM, and late-phase elevation of eosinophils in bronchoalveolar lavage fluid (Table 1).

RIP parameters indicative of TAA were validated by simultaneous RIP and conventional determination of $\mathrm{R}_{\mathrm{L}}$ and $\mathrm{C}_{\mathrm{dyn}}$. In an initial session, spontaneously breathing animals were challenged with increasing cumulative doses of aerosolized methacholine to induce graded changes in $\mathrm{R}_{\mathrm{L}}$ and $\mathrm{C}_{\mathrm{dyn}}$. Four weeks later, bronchoconstriction was induced with aerosolized HDM allergen, and then reversed by aerosolized albuterol. Correlations between RIP parameters, $\mathrm{R}_{\mathrm{L}}$, and $\mathrm{C}_{\mathrm{dyn}}$ were then determined.

Following the validation phase of the study, nocturnal responses to daytime allergen challenge were investigated. Animals were challenged with aerosolized saline or HDM allergen early in the morning. TAA was then monitored by RIP throughout the rest of the day and overnight.

\section{Animals}

Over a 10-year period prior to the study, 38 Cynomolgus macaques were obtained from Alpha Genesis, Inc.

Table I Characteristics of nonallergic and allergic groups

\begin{tabular}{|c|c|c|c|c|c|c|}
\hline & $\begin{array}{l}\text { Serum } \alpha \text {-HDM-IgE } \\
\text { (\% allergic control) }\end{array}$ & $\begin{array}{l}\text { Eosinophils } \\
\text { in BAL (\%) }\end{array}$ & $\begin{array}{l}\text { Total cells in BAL } \\
\text { compartment } \times 10^{6}\end{array}$ & $\begin{array}{l}\text { HDM concentration } \\
\text { (AU/mL) }\end{array}$ & $\Delta \mathbf{R}_{\mathrm{L}}(\%)$ & $\Delta \mathrm{C}_{\mathrm{dyn}}(\%)$ \\
\hline Nonallergic & $15.8 \pm 3.5$ & $\mathrm{I} \pm 0.4$ & $9.58 \pm 3.47$ & $2500 \pm 0$ & $0.6 \pm 5.3$ & $-5.6 \pm 4.9$ \\
\hline Allergic & $70.4 \pm 5.1$ & $35.1 \pm 4.7$ & $13.65 \pm 1.76$ & $360 \pm 120.7$ & $103.4 \pm 16.7$ & $-47.6 \pm 3.4$ \\
\hline$P$ value & $<0.0001$ & $<0.0001$ & 0.236 & $<0.0001$ & $<0.0001$ & $<0.0001$ \\
\hline
\end{tabular}

Notes: Values are mean $\pm S E$, nonallergic, $n=11$, allergic, $n=21$. Animals were exposed to increasing concentrations of HDM in sterile saline until the concentration presented in this table elicited the associated $\Delta \mathrm{R}_{\mathrm{L}}$ or $\Delta \mathrm{C}_{\mathrm{dyn}}$ during $\mathrm{I} 5$ minutes following the HDM challenge.

Abbreviations: BAL, in bronchoalveolar lavage; HDM, house dust mite; $\mathrm{C}_{\mathrm{dyn}}$, dynamic compliance; $\mathrm{R}_{\mathrm{L}}$, lung resistance; $\mathrm{SE}$, standard error of measurement. 
(Yemassee, SC) and sensitized to HDM as described previously. ${ }^{33,34}$ Briefly, the animals were sensitized by intraperitoneal injections of Dermatophagoides pteronyssinus and Dermatophagoides farinae extract adsorbed to Alum (156 units of each allergen [Greer Laboratories, Lenoir, NC]; Imject Alum [Pierce, Rockford, IL]) at 2-4 week intervals for six months. Chronic airway disease was induced by subsequent periodic exposure to HDM aerosol at 4-6 week intervals. At the time of the study, the animals ranged in age from 3.6 to 9.4 years, weighed 2.3 to $7.5 \mathrm{~kg}$, and had exhibited allergic asthma symptoms for $1-8$ years.

The animals were group-housed in accordance with US Department of Agriculture guidelines within a BSL2 facility at East Carolina University, which is fully accredited by the Association for the Assessment and Accreditation for Laboratory Animal Care, International. All protocols were approved by the Institutional Animal Care and Use Committee of East Carolina University.

Exposure to allergen between intentional provocations was minimized by HEPA (high efficiency particulate air) filtration of all air circulating through the animal rooms, rinsing the rooms and cages with pressurized water twice each day, and running the cages through an automated washer weekly. All personnel entering the rooms wore appropriate personal protective gear.

\section{Enzyme-linked immunosorbent assay for allergen-specific lgE}

Serum levels of HDM-specific IgE were measured as described previously. ${ }^{33}$ Ninety-six well plates were coated with HDM allergen $\left(10 \mu \mathrm{g} / \mathrm{mL}\right.$ per well, incubated overnight at $4^{\circ} \mathrm{C}$ in $0.01 \mathrm{M}$ sodium bicarbonate buffer, $\mathrm{pH}$ 9.6). The plates were blocked with $10 \%$ fetal bovine serum; $100 \mu \mathrm{L}$ of serum $(1: 100$ dilutions) was added to the wells. The plates were incubated overnight at $4^{\circ} \mathrm{C}$. IgE was detected by incubating with $1 \mu \mathrm{g} / \mathrm{mL}$ biotinylated goat anti-human IgE (Vector Lab, Burlingame, CA) for one hour at room temperature, and then developing with streptavidin-conjugated horseradish peroxidase (BD PharMingen, San Diego, CA) and 3,3',5,5'-tetramethylbenzidine (BD PharMingen). The reaction was stopped by addition of sulfuric acid, and the absorbance at $450 \mathrm{~nm}$ was determined. Relative IgE titers were determined by comparison with a serum sample pooled from a group of highly allergic animals.

\section{Determination of lung resistance and dynamic compliance}

Animals were anesthetized with Telazol ${ }^{\circledR} 2.0 \mathrm{mg} / \mathrm{kg}$, intramuscularly, stabilized on propofol (10 to $15 \mathrm{mg} / \mathrm{kg} / \mathrm{hour}$, intravenously), and intubated. $\mathrm{R}_{\mathrm{L}}, \mathrm{C}_{\mathrm{dyn}}$ and respiratory rate (RR) were measured by a conventional pressure-flow technique. ${ }^{33,34}$ Tracheal airflow was measured using a heated Fleisch Pneumotachograph (size 00, Fleisch, Lausanne) connected to a Validyne pressure transducer (Model DP 45-14, Validyne Engineering, Northridge, CA). Intrathoracic pressure was measured using an esophageal balloon connected to a Model DP 45-24 Validyne pressure transducer. Flow and pressure signals were analyzed using a MuMed PR800 lung function recorder (MuMed, London). Baselines of $\mathrm{R}_{\mathrm{L}}, \mathrm{C}_{\mathrm{dyn}}$, and RR were determined during a one-minute period of quiet breathing. Subsequent responses to aerosolized saline (vehicle), methacholine, HDM, and albuterol were expressed as raw data or as a percentage change from baseline or saline as appropriate.

\section{House dust mite challenge}

Baseline pulmonary function was recorded. The animals were challenged for four minutes with nebulized sterile saline delivered through the endotracheal tube (Devilbiss Ultrasonic Nebulizer, SUN-99HD, Red Lion, PA or Nouvag Ultrasonic 2000, Nouvag). Condensation on the inner surface of the endotracheal tube was cleared by aspiration, and pulmonary function was monitored for one minute. The challenge was repeated with increasing concentrations of nebulized HDM allergen (1, 10, 100, $500,2500 \mathrm{AU} / \mathrm{mL}$ ) until a $100 \%$ increase in $\mathrm{R}_{\mathrm{L}}$ or a $50 \%$ decrease in $\mathrm{C}_{\mathrm{dyn}}$ was observed. ${ }^{33}$ Once the target change in $\mathrm{R}_{\mathrm{L}}$ or $\mathrm{C}_{\text {dyn }}$ was observed, pulmonary function was monitored at five-minute intervals for 15 minutes. Following the last recording, the animals were treated with nebulized albuterol sulfate (four minutes, $0.083 \%$ solution, Ventolin Nebules, Orlando, FL).

Arterial oxygen saturation was monitored by pulse oximetry (SurgiVet Model V3304, Harvard Apparatus, Holliston, MA or Cardell ${ }^{\circledR}$ Model 9403 and 9405, Sharn Veterinary Inc., Tampa, FL), and supplemental $\mathrm{O}_{2}$ was delivered as needed to maintain $\mathrm{O}_{2}$ saturation above $70 \%$.

\section{Methacholine challenge}

Animals were anesthetized and instrumented as described earlier. Baseline pulmonary function was recorded, and sterile saline was delivered for two minutes. Condensation on the tube was cleared by aspiration, and pulmonary function was monitored for one minute. Cumulative doses of methacholine (A2251, Sigma, St Louis, MO) between $0.001 \mathrm{mg} / \mathrm{mL}$ and $10 \mathrm{mg} / \mathrm{mL}$ were delivered until a $100 \%$ increase in $\mathrm{R}_{\mathrm{L}}$ and/or a $50 \%$ decrease in $\mathrm{C}_{\text {dyn }}$ was observed. ${ }^{33}$ 
The range of aerosolized methacholine doses was based on previous experience with this model, ${ }^{33}$ published results from tissue bath experiments, ${ }^{37,38}$ and empiric observations with this cohort of animals. A methacholine concentration of $0.001 \mathrm{mg} / \mathrm{mL}$ did not induce bronchoconstriction, whereas concentrations greater than $10 \mathrm{mg} / \mathrm{mL}$ induced high levels of secretions in the airways. Therefore, $10 \mathrm{mg} / \mathrm{mL}$ methacholine was chosen as the upper level. The provocative concentration of methacholine leading to a 50\% maximal response $\left(\mathrm{PC}_{50}\right)$ for $\mathrm{R}_{\mathrm{L}}, \mathrm{C}_{\mathrm{dyn}}$, and $\mathrm{RR}$ was calculated for individual animals, and then mean $\mathrm{PC}_{50}$ was determined for all animals.

\section{Endotracheal bronchoalveolar lavage}

Twenty-four hours after HDM challenge, the animals were anesthetized with Telazol and propofol, then intubated. A sterile $2.5 \mathrm{~mm}$ OD Tygon tube was wiped with Surgilube ${ }^{\circledR}$, advanced through the endotracheal tube, and gently wedged into an airway. The lung segment beyond the tip of the catheter was lavaged with $7 \mathrm{~mL}$ of sterile saline, which was collected by gentle aspiration. Total cell counts were determined using a Coulter Counter (Beckman Coulter Instruments, Miami, FL). Slides were generated using a Cytospin and stained with DiffQuik (VWR Scientific, So. Plainfield, NJ). Manual differential cell counts were acquired by counting 200 cells per sample.

\section{Respiratory inductive plethysmography}

RIP parameters were measured using the LifeShirt System (Vivometrics, Inc., Ventura, CA). The LifeShirt incorporated two parallel sinusoidal wire arrays encircling the thorax and abdomen. A small alternating current was passed through the wires, inducing a magnetic field. Changes in the shape of the arrays during breathing altered the magnetic field, inducing a current that was detected as a change in the frequency of the applied current, which translated into lung volumes and flows on a breath-by-breath basis through coefficients obtained during calibration procedures. Changes in animal posture and activity were monitored by accelerometers in the jackets allowing movement artifacts to be filtered out of the dataset. ${ }^{39-41}$

Animals were fitted with the LifeShirt jackets such that the rib cage band was positioned over the sternum and the abdominal band positioned immediately cephalad to the pelvic girdle. ${ }^{41}$ The circumference of each band was adjusted to provide rib cage and abdomen deflections of 20 to 200 assumed milliliters (AmL) prior to calibration. The LifeShirt jackets were fitted on the animals before any airway challenges began and were left on for about 24 hours. Raw data records were analyzed with VivoLogic software (Vivometrics, Inc.). The default settings were overridden to permit analysis of the small tidal volumes $(100 \%$ tidal volume $=40 \mathrm{AmL}$; minimum acceptable tidal volume $=2.5 \%$; minimum acceptable effort volume $=2.5 \%$ ). A fixed volume calibration was then performed using the tidal volumes recorded by the MuMed lung function recorder during the one-minute baseline period. ${ }^{42,43}$ The calibrated records were used to determine the RIP parameters (expressed as calibrated raw data or as a percentage change from baseline, see below) to be compared with simultaneously determined conventional measures of $\mathrm{R}_{\mathrm{L}}$ and $\mathrm{C}_{\mathrm{dyn}}$.

Three RIP-derived parameters analyzed by the LifeShirt software were used as indicators of TAA, ie, phase angle (PhAng) of the abdomen and rib cage waveforms, baseline effort phase relation (eBPRL), and effort phase relation (ePhRL). PhAng is a common parameter used to compare the initial slopes of two sinusoidal waveforms. ${ }^{44}$ As abdominal and thoracic movement become asynchronous, $\mathrm{PhAng}$ increases. Effort parameters provide an indication of the amount of time during a breath that deflection of the abdomen and chest wall are asynchronous (ie, the two are moving in opposite directions instead of in unison). eBPRL is the percentage of time during inspiration that the rib cage is moving in the opposite direction from the abdomen, and ePhRL is the percentage of time during the total breath that the rib cage and abdomen are moving in opposite directions. Thus, TAA is associated with increases in PhAng, eBPRL, and ePhRL.

\section{Nocturnal study}

Following HDM provocation, the animals were recovered from anesthesia and returned to their cages. RIP readouts were monitored continuously until the next morning. The lower threshold for movement artifact was set as the maximum acceleration values associated with quiet breathing for each animal while under anesthesia. All records associated with accelerations greater than this maximum value were removed from the dataset.

Thirty-minute averages of eBPRL and ePhRL (but not $\mathrm{PhAng}$, see Results section) were plotted from $1 \mathrm{pm}$ the day of HDM provocation until 6 am the next day. A 30-minute period between $1 \mathrm{pm}$ and $10 \mathrm{pm}$ was identified during which there was little animal movement and low incidence of TAA (ie, minimum average eBPRL or ePhRL for the period, labeled as baseline in Figure 4). This relatively normal, quiet breathing period served as a baseline for the nocturnal 
studies. eBPRL and ePhRL were plotted against time and the area under the curve (AUC) between $10 \mathrm{pm}$ the day of HDM challenge and 5 am the next day was determined using SigmaPlot Version 10 (Systat Software, Inc., San Jose, CA, see Figure 4). The AUC was used as an indicator of TAA incidence and to compare nocturnal responses among subjects.

\section{Statistics}

Recorded respiratory parameters were expressed as calibrated raw data or as a percentage change from the baseline control period. The relationships between $\mathrm{R}_{\mathrm{L}}, \mathrm{C}_{\mathrm{dyn}}, \mathrm{RR}$, and RIP parameters were investigated by simple and multiple linear regressions using SPSS software (SPSS, Inc., Chicago, IL). The methacholine $\mathrm{PC}_{50}$ of each parameter was determined by Hill fit analysis for each animal (Systat Software, Inc., San Jose, CA). Differences between two means were assessed by Student's t-test for independent observations $(P \leq 0.05)$. Differences between three or more means were assessed by one-way analysis of variance (ANOVA) with Tukey HSD post hoc test $(P \leq 0.05)$. Values are reported as mean \pm standard error of measurement.

\section{Results}

\section{Methacholine and HDM challenges}

To validate RIP as a measure of TAA associated with underlying airway responses, the relationships between $R_{L}, C_{d y n}$, and RIP parameters indicative of TAA were defined under controlled conditions where methacholine and HDM were used to induce acute bronchoconstriction, and albuterol to induce bronchodilation.

Nonallergic animals had minimal changes in all parameters at the highest concentration of methacholine (data not shown), whereas in allergic animals the highest concentration of methacholine increased $\mathrm{RR}$ and $\mathrm{R}_{\mathrm{L}}$ and decreased $\mathrm{C}_{\text {dyn }}$. $R R$ exhibited a higher sensitivity to methacholine than $C_{\text {dyn }}$ and $R_{L}$, while $C_{d y n}$ exhibited sensitivity to methacholine that was intermediate between $R R$ and $R_{L}$ (Table 2). These results were consistent with independent modulation of $R R$ and $R_{L}$, and with changes in $\mathrm{C}_{\mathrm{dyn}}$ that were due to reduced alveolar filling time as RR increased. Multiple linear regression of values recorded before and after methacholine challenge revealed significant associations of $\mathrm{R}_{\mathrm{L}}$ and $\mathrm{C}_{\text {dyn }}$ with eBPRL, ePhRL and PhAng (eBPRL: $r=0.49, P<0.05$; ePhRL: $r=0.41$, $P<0.05$; PhAng: $r=0.45, P<0.05$ ).

At a later date, the animals were challenged with aerosolized HDM antigen to induce acute bronchoconstriction, and were then treated with albuterol to reverse the
Table 2 Methacholine $\mathrm{PC}_{50}$ of conventional respiratory indexes

\begin{tabular}{|c|c|c|c|c|c|}
\hline & \multicolumn{2}{|c|}{$\begin{array}{l}\text { Methacholine } \\
\mathrm{PC}_{50}(\mathrm{mg} / \mathrm{mL})\end{array}$} & \multicolumn{3}{|c|}{$P$ value } \\
\hline & Mean & SE & $\mathbf{R R}$ & $C_{d y n}$ & $\mathbf{R}_{\mathrm{L}}$ \\
\hline$\overline{R R}$ & 0.07 & 0.01 & - & 0.031 & 0.001 \\
\hline$C_{d y n}$ & 0.23 & 0.05 & 0.031 & - & 0.001 \\
\hline$R_{L}$ & 0.63 & 0.09 & 0.001 & 0.001 & - \\
\hline
\end{tabular}

Abbreviations: $R R$, respiratory rate; $C_{d y n}$, dynamic compliance; $R_{L}$, lung resistance; $\mathrm{SE}$, standard error of measurement.

HDM-induced bronchoconstriction. When the effects of HDM and albuterol were separated, the RIP parameters tracked closely with changes in $\mathrm{C}_{\text {dyn }}$ and $\mathrm{R}_{\mathrm{L}}$ (Figure 1 ). Although the directions of $\mathrm{C}_{\text {dyn }}$ and $\mathrm{R}_{\mathrm{L}}$ were opposite, the relative magnitude of HDM-induced changes were similar $\left(\Delta \mathrm{C}_{\mathrm{dyn}}: P=0.0001\right.$ and $\Delta \mathrm{R}_{\mathrm{L}}: P=0.001, \mathrm{HDM}$ versus saline) and both parameters exhibited at least partial reversal with albuterol (Figures 1B and 1C). HDM provoked increases in eBPRL, ePhRL, and PhAng $(P<0.01$, HDM versus saline) that were reversed by albuterol (Figures 1D-1F). In contrast, RR increased with $\operatorname{HDM}(P<0.001$, HDM versus saline), but did not reverse with albuterol (Figure 1A). These results demonstrated that TAA, as measured by changes in RIP, coincided with changes in $\mathrm{R}_{\mathrm{L}}$ and $\mathrm{C}_{\mathrm{dyn}}$ induced by diverse agents.

The aggregate HDM and albuterol data were analyzed by multiple linear regression, which revealed associations of $\mathrm{R}_{\mathrm{L}}$ and $\mathrm{C}_{\text {dyn }}$ with eBPRL, ePhRL, and PhAng as described by the following equations (See Table 3 ):

$$
\begin{gathered}
\text { eBPRL }=26.8-3.8 \mathrm{C}_{\text {dyn }}+1.7 \mathrm{R}_{\mathrm{L}} \\
\mathrm{ePhRL}=30.2-4.4 \mathrm{C}_{\text {dyn }}+1.7 \mathrm{R}_{\mathrm{L}} \\
\text { PhAng }=98.6-11.4 \mathrm{C}_{\text {dyn }}+2.1 \mathrm{R}_{\mathrm{L}}
\end{gathered}
$$

Thus, the RIP parameters increased as $\mathrm{C}_{\text {dyn }}$ decreased and, independently, after adjustment for $\mathrm{C}_{\mathrm{dyn}}$, the RIP parameters increased as $R_{L}$ increased. The regression equations were consistent with an inverse relation between $\mathrm{R}_{\mathrm{L}}$ and $\mathrm{C}_{\mathrm{dyn}}$. This relationship was verified by plotting coincident values of $R_{L}$ against $\mathrm{C}_{\text {dyn }}$ for data collected during both the methacholine and HDM challenges. The resulting curve exhibited an inverse first-order relationship $(r=0.69, P<0.05$ by simple linear regression of $1 / \mathrm{R}_{\mathrm{L}}$ versus $\mathrm{C}_{\mathrm{dyn}}$ ).

\section{Nocturnal asthmatic response to house dust mite challenge}

In follow-up experiments to those discussed above, animals were challenged with aerosol HDM between 6 am and $10 \mathrm{am}$, and then acute bronchoconstriction was reversed with albuterol. The animals were recovered from anesthesia, and returned to their cages. Excursions of the rib cage 
A

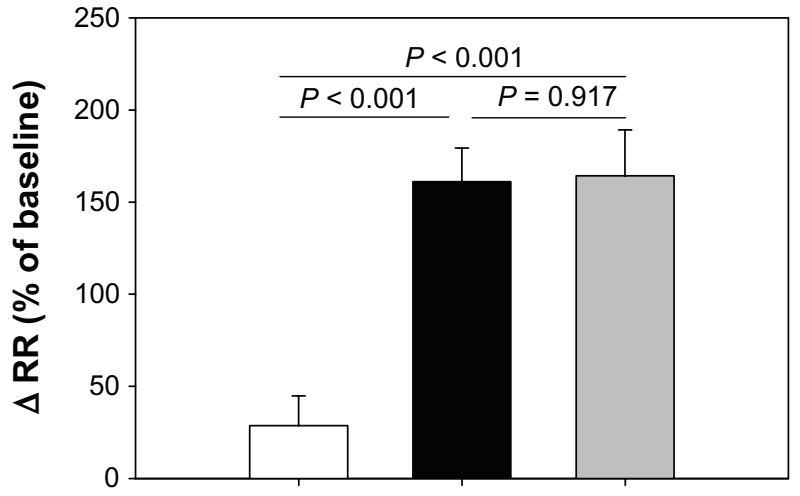

B

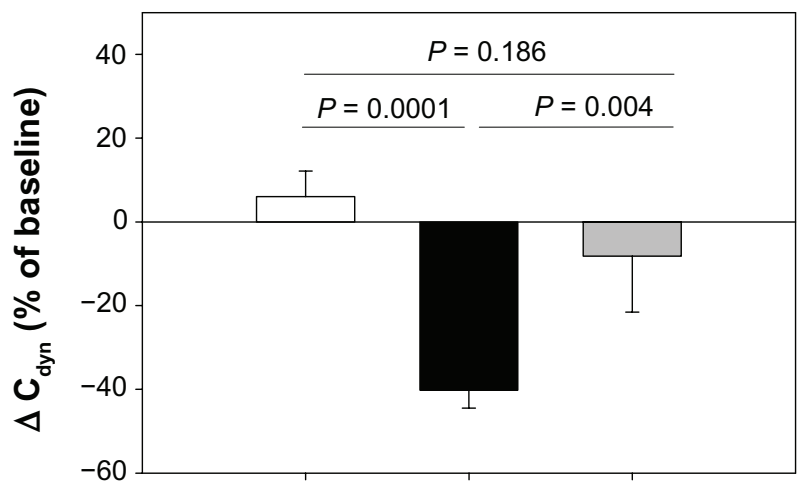

C

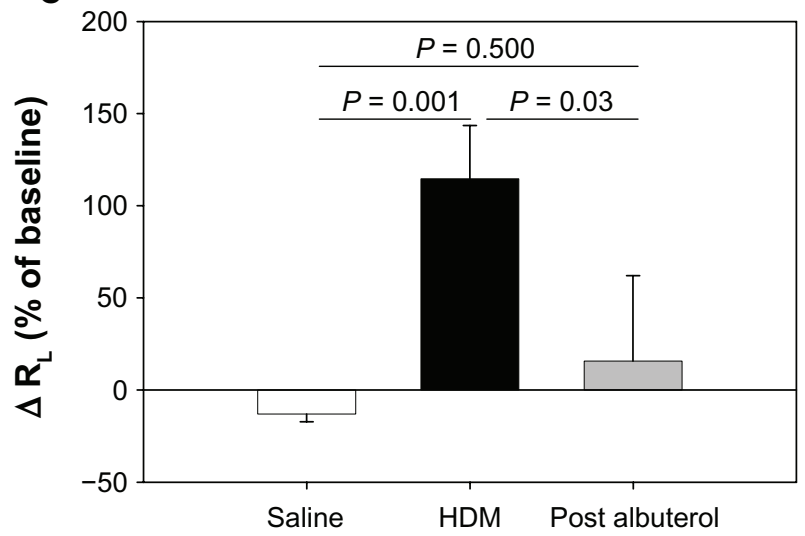

D

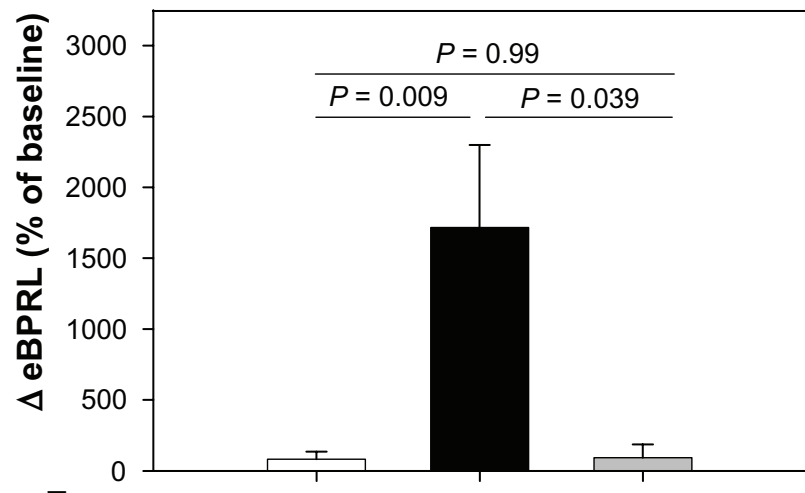

E

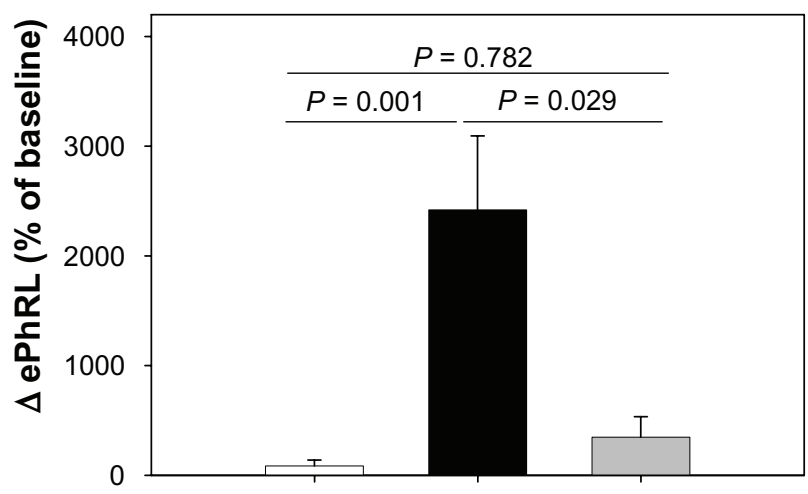

$\mathbf{F}$

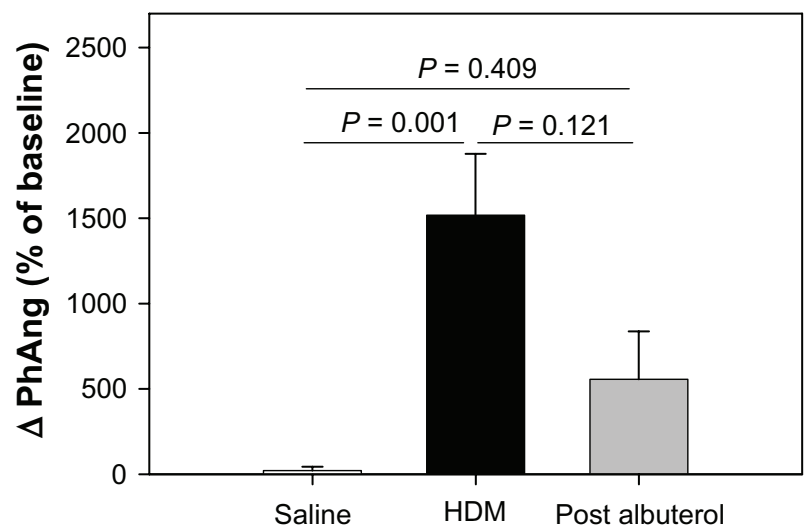

Figure I Responses to sequential exposure of aerosolized saline, maximum concentration of HDM extract, and albuterol. Shown are changes in (A) RR, (B) $C_{d y n}$, (C) $R_{L}$, (D) eBPRL, (E) ePhRL, and (F) PhAng. Responses to saline and HDM were recorded in 23 animals. Albuterol was administered to eight animals exhibiting severe and prolonged increases in airway resistance. The changes were expressed as a percentage of the baseline measurement recorded prior to saline delivery. Values are expressed as means \pm SE. Abbreviations: HDM, house dust mite; $R R$, respiratory rate; $C_{d y n}$, dynamic compliance; $R_{L}$, lung resistance; eBPRL, baseline effort phase relation; ePhRL, effort phase relation; PhAng, phase angle; SE, standard error of measurement.

and the abdomen were monitored continuously until the next morning. Figure 2 shows representative RIP recordings for one animal while anesthetized during the baseline and the early asthmatic response (Figure 2, left panels), and later while conscious during the afternoon or evening baseline and the nocturnal asthmatic response (Figure 2, right panels). PhAng (calculated from the Konno-Mead diagram) has been used in previous studies to assess TAA. ${ }^{15,41,44}$ However, PhAng is affected by the shape of the abdominal and ribcage waveforms, as in Tables and other figures with a triangular waveform being associated with increased error. ${ }^{15,41}$ Triangular waveforms were commonly observed in the current study (Figure 2) and, therefore, PhAng was not used as an indicator of TAA at night. The effort parameters, eBPRL and ePhRL, correlated well with labored breathing exhibited during the acute response to HDM and methacholine provocation, and were therefore used to monitor for nocturnal respiratory disturbances. 
Table 3 Multiple linear regression results of eBPRL, ePhRL, and $\mathrm{PhAng}$ recorded during the first 30 minutes following HDM challenge

\begin{tabular}{|c|c|c|c|c|c|}
\hline \multirow[t]{2}{*}{$\begin{array}{l}\text { Dependent } \\
\text { variable }\end{array}$} & \multirow[t]{2}{*}{$\begin{array}{l}\text { Independent } \\
\text { variable }\end{array}$} & \multicolumn{2}{|c|}{$\begin{array}{l}\text { Regression } \\
\text { coefficients }\end{array}$} & \multirow[t]{2}{*}{$\mathbf{t}_{\mathrm{bj}}$} & \multirow[t]{2}{*}{$P$} \\
\hline & & $B_{j}$ & $S E s_{b j}$ & & \\
\hline \multirow[t]{3}{*}{ eBPRL } & Intercept & 26.80 & 13.72 & 1.95 & 0.056 \\
\hline & $C_{\text {dyn }}$ & -3.85 & 1.25 & -3.08 & 0.003 \\
\hline & $R_{L}$ & 1.65 & 0.41 & 4.08 & $<0.001$ \\
\hline \multirow[t]{3}{*}{ ePhRL } & Intercept & 30.15 & 13.99 & 2.16 & 0.035 \\
\hline & $C_{d y n}$ & -4.43 & 1.27 & -3.48 & 0.001 \\
\hline & $R_{L}$ & 1.68 & 0.41 & 4.06 & $<0.001$ \\
\hline \multirow[t]{3}{*}{ PhAng } & Intercept & 98.62 & 25.20 & 3.91 & $<0.001$ \\
\hline & $C_{d y n}$ & -11.42 & 2.29 & -4.98 & $<0.001$ \\
\hline & $R_{L}$ & 2.07 & 0.74 & 2.782 & 0.007 \\
\hline
\end{tabular}

Note: The $P$ value is a test of $H_{0}: \beta=0$ against $H_{2}: \beta \neq 0$.

Abbreviations: HDM, house dust mite; $R R$, respiratory rate; $C_{d y n}$, dynamic compliance; $R_{L}$, lung resistance; eBPRL, baseline effort phase relation; ePhRL, effort phase relation; PhAng, phase angle; SE, standard error or measurement.

To assess the long-term stability of eBPRL and ePhRL, both parameters were recorded in nonallergic animals overnight. No significant change was observed in eBPRL and ePhRL over the nocturnal time period between $10 \mathrm{pm}$ and 5 am (Figure 3), whereas in most allergic animals, eBPRL and $\mathrm{ePhR}$ gradually increased from the baseline, reaching a peak value around 3:30 am (Figure 4). To quantify nocturnal TAA, we determined the AUC between $10 \mathrm{pm}$ and 5 am and determined the 99\% confidence intervals for nonallergic animals (dashed line in Figure 5A and B). Plots of eBPRL and ePhRL against time for allergic animals revealed 15 of 21 animals with AUCs greater than the upper boundary of the $99 \%$ confidence interval. These 15 animals were therefore classified as nocturnal asthmatic responders (NARs). The allergic group mean AUCs for changes in eBPRL and ePhRL were greater than the nonallergic control group mean AUCs (Figure $5 \mathrm{~A}$ and $\mathrm{B}, \triangle \mathrm{eBPRL}$ : $P<0.001, \Delta$ ePhRL: $P=0.001)$. In control experiments, the NAR group did not exhibit nocturnal responses following challenges between 6 am and 10 am with aerosolized saline alone (data not shown).

To evaluate the reproducibility of the nocturnal response, eight animals exhibiting acute responses to HDM were rechallenged at a later date (Figure 6). Six of the animals had been classified as NARs in the previous trial (shown as six symbols above the dashed lines for challenge number 1, Figure 6) and two animals had not been classified as NARs. Four of these six NARs from the first trial exhibited nocturnal responses following the second challenge (Figure 6). Both the animals that had not been classified as NARs in the previous trial exhibited nocturnal responses for the second challenge. Conversely, two animals that exhibited a nocturnal response following the first challenge exhibited minimal increase in eBPRL and ePhRL following the second challenge. Manifestation of a nocturnal response and its magnitude could not be attributed to the provocative concentration of HDM used to elicit the early-phase response or the magnitude of the change in $\mathrm{R}_{\mathrm{L}}$ and $\mathrm{C}_{\text {dyn }}$ during the early-phase response (data not shown).

\section{Discussion}

In spontaneously breathing animals, large and small airway constriction manifest as changes in resistance and compliance. These changes may contribute to TAA which results from inability of thoracic volume to change in phase with abdominal volume due to restricted airflow through the lungs. To our knowledge, this is the first report on the use of RIP to measure TAA in a NHP model of allergic asthma and to apply the technique to document nocturnal responses in animals following allergen challenge. TAA as indicated by RIP eBPRL and ePhRL parameters correlated with changes in $\mathrm{C}_{\text {dyn }}$ and $\mathrm{R}_{\mathrm{L}}$ in Cynomolgus macaques during bronchoconstriction induced by methacholine or HDM, and bronchodilation induced by albuterol. Following HDM challenge in the early morning, TAA was shown to increase, with a peak between 3 am and 4 am the next morning. The nocturnal response exhibited intra-animal variability in both incidence and magnitude, which is consistent with manifestation of nocturnal asthma symptoms in humans.

RIP has been used extensively to assess apnea and breathing patterns during sleep in humans ${ }^{20,21,23,24,26}$ and animals. ${ }^{45}$ Multiple human studies have demonstrated correlations among TAA, bronchoconstriction, and bronchodilation. ${ }^{15,25,44}$ In contrast with TAA, other RIP parameters, particularly ventilation, do not appear to be useful in assessing pulmonary function in asthmatic subjects, ${ }^{19,46}$ possibly due to artifact induced in volume measurements by asynchronous movement of the chest wall and abdomen during bronchoconstriction. ${ }^{47}$ In this study of allergic NHP, TAA as measured by RIP correlated with changes in $\mathrm{R}_{\mathrm{L}}$ and $\mathrm{C}_{\text {dyn }}$ during HDM- and methacholine-induced bronchoconstriction. In contrast, a previous study in foals did not observe these correlations during histamine-induced bronchoconstriction. ${ }^{12}$ The reason for this discrepancy is not clear.

In asthmatics there is a high prevalence of nocturnal symptoms, ${ }^{2,3}$ with peak pulmonary resistance ${ }^{3}$ and airway 


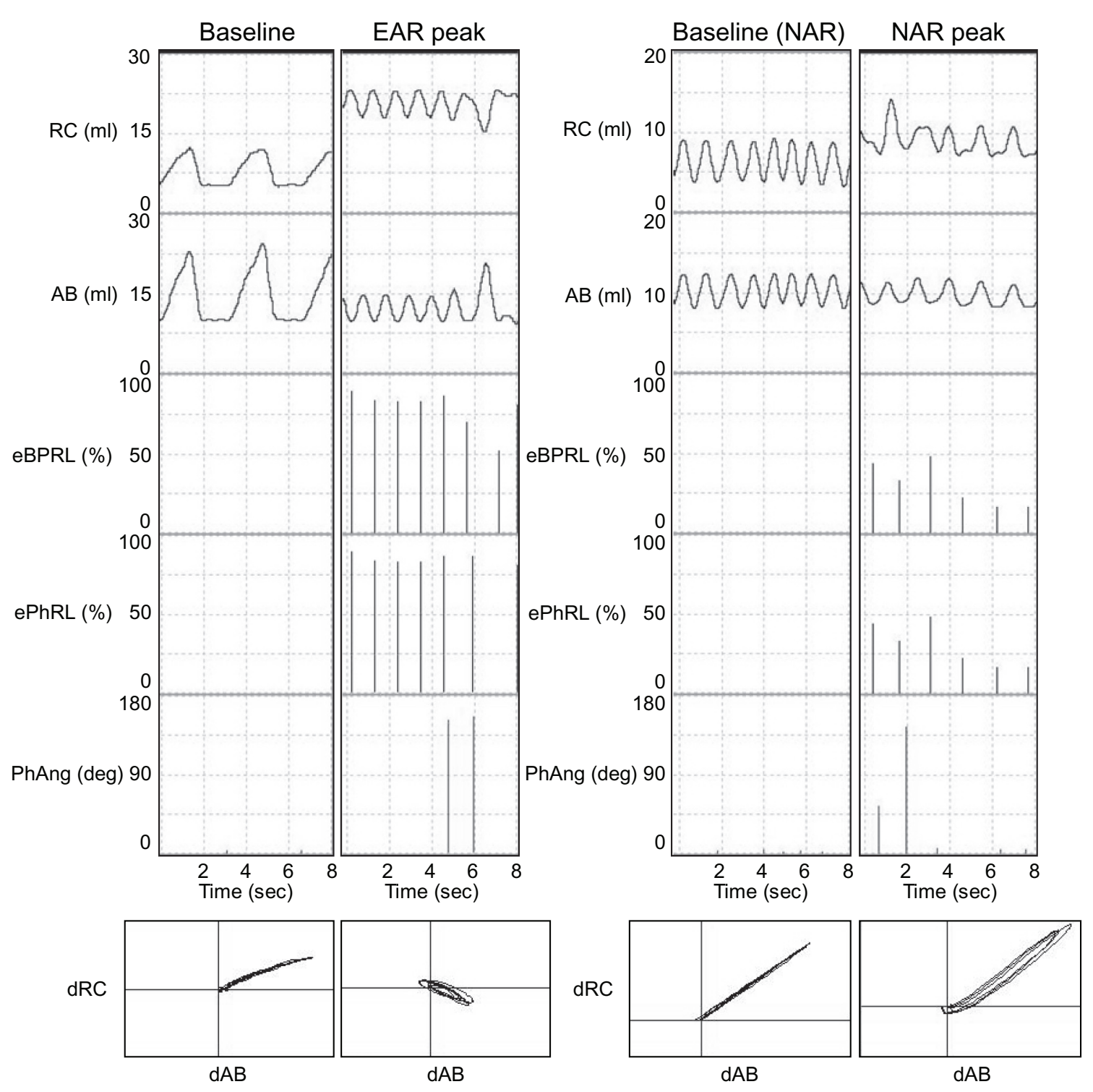

Figure 2 Representative RIP recordings of one animal (ID: 8065) during both the early asthmatic response and the nocturnal asthmatic response. Excursions of the rib cage (RC) and the abdomen (AB), as well as corresponding eBPRL, ePhRL, and PhAng are shown for multiple breaths. Note the triangular RC and $A B$ wave forms, and that eBPRL and ePhRL are better reflections of TAA than PhAng during the early asthmatic response peak and also are good reflections of TAA during the nocturnal asthmatic response peak. The Konno-Mead diagram (bottom of each vertical panel) shows the direction of RC movement ( $d R C$ ) and the direction of $A B$ movement ( $A B$ ) during inhalation and exhalation in three breaths. Abbreviations: $A B$, abdomen; RC, rib cage; eBPRL, baseline effort phase relation; ePhRL, effort phase relation; PhAng, phase angle; TAA, thoracoabdominal asynchrony.

inflammation ${ }^{4,5}$ observed around $4 \mathrm{am}$. In this study of allergic NHP, both eBPRL and ePhRL gradually increase during the night following an early morning HDM challenge, which is consistent with the time course of nocturnal symptoms in humans. These nocturnal increases in TAA may be linked to underlying bronchoconstriction, but this cannot be proven without overt intervention at night. TAA is also impacted by changes in respiratory muscle load, control of auxiliary muscles of breathing, and respiratory muscle dysfunction. ${ }^{22}$ These factors cannot be excluded as underlying causes of nocturnal TAA.
Regardless, the data provide compelling evidence that daytime exposure to allergen results in nocturnal disturbances in breathing.

The incidence and severity of nocturnal asthma symptoms in humans is variable, both between and within subjects. ${ }^{7,48}$ Likewise, nocturnal TAA following HDM challenge in allergic NHP was variable between animals and within individual animals over time. Shigemitsu et a ${ }^{49}$ reported that $60 \%$ of asthmatic subjects manifest nocturnal symptoms. This number compares favorably with the $71 \%$ incidence observed in the current study. Fix 


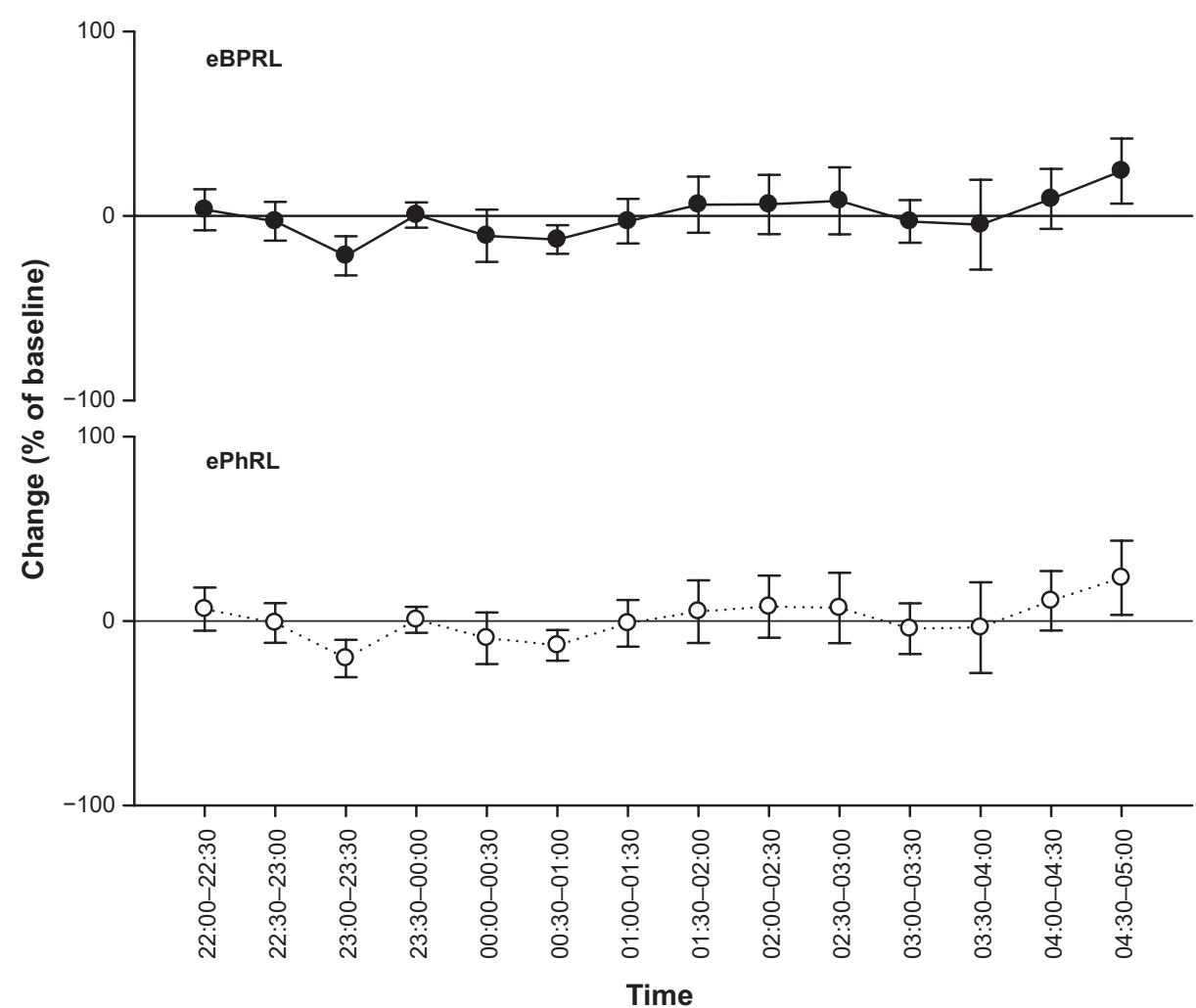

Figure 3 Long-term stability of nocturnal eBPRL and ePhRL. Plots of eBPRL and ePhRL (30-minute averages) starting at 10 pm and ending at 5 am next morning in II nonallergic animals. The changes were expressed as a percentage of the baseline measurement recorded during afternoon. Values are means $\pm S E$ at each time point. Abbreviations: eBPRL, baseline effort phase relation; ePhRL, effort phase relation; SE, standard error or measurement.

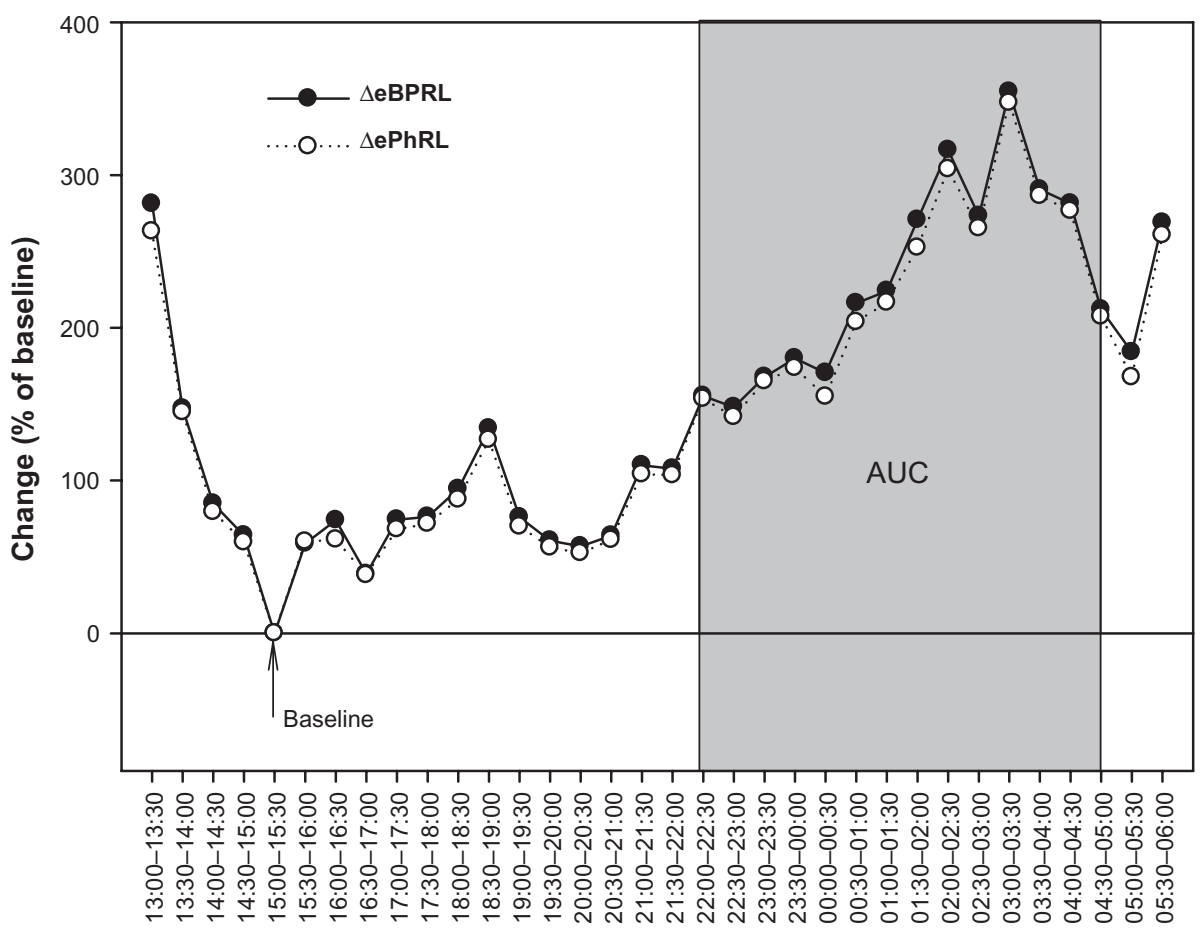

Time

Figure 4 Representative nocturnal eBPRL and ePhRL response of the same animal represented in Figure 2 (ID: 8065). Plot of eBPRL (filled circle) and ePhRL (open circle) (30-minute averages) starting at I pm and ending at 6 am next morning. AUC was started at $10 \mathrm{pm}$ and ended at 5 am.

Abbreviations: eBPRL, baseline effort phase relation; ePhRL, effort phase relation; AUC, area under the curve. 

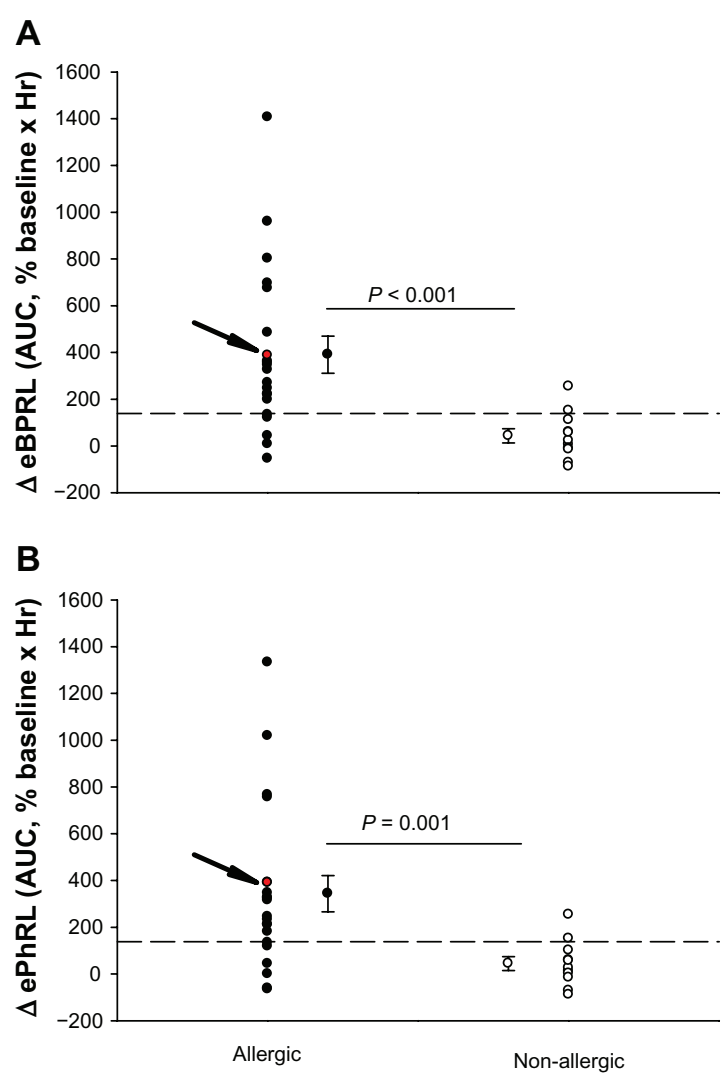

Figure 5 Changes in (A) eBPRL and (B) ePhRL between $10 \mathrm{pm}$ and 5 am for each individual animal in both the allergic group $(n=2 I)$ and the nonallergic group $(n=I I)$. $A$ and $B$ the allergic group mean $A \cup C$ for eBPRL and $e P h R L$ were significantly greater than the nonallergic control group mean AUC. The arrow indicates values for animal ID: 8065 which is also represented in Figure 2 and 4. Dashed lines represent the upper limit of the $99 \%$ confidence interval for the nonallergic group.

Abbreviations: eBPRL, baseline effort phase relation; ePhRL, effort phase relation; $A \cup C$, area under the curve.

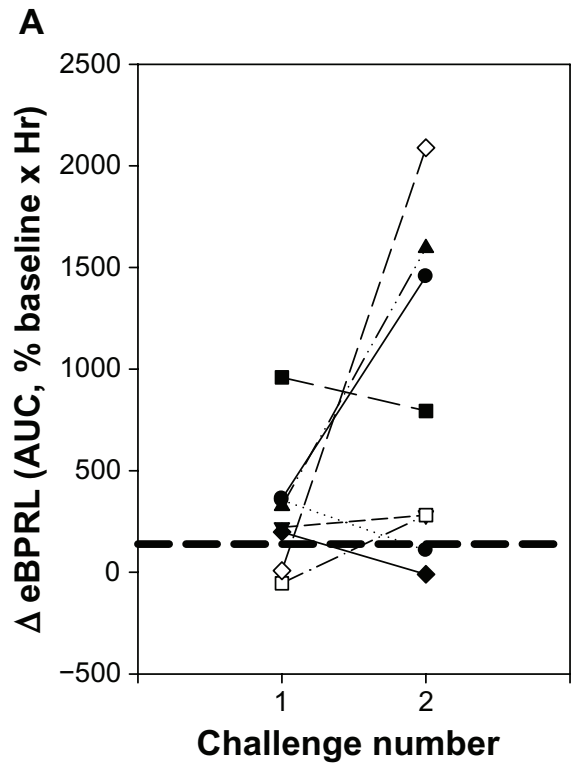

et al reported that occurrence of nocturnal symptoms is linked to the severity of asthma, ${ }^{50}$ but other investigators fail to substantiate a definitive link between any particular phenotypic feature of early acute allergic responses and late asthmatic responses in humans and animals, ${ }^{51-53}$ including the occurrence of nocturnal symptoms. ${ }^{7}$ In the NHP model, nocturnal TAA could not be attributed to any of the functional parameters measured during the early asthmatic response, including the provocative concentration of HDM or the magnitude of the acute bronchoconstriction.

\section{Conclusion}

In summary, RIP parameters indicative of TAA correlated with decrease in pulmonary function during methacholine challenge and the early asthmatic response in HDM-sensitive Cynomolgus macaques. Using RIP as a measure of TAA revealed nocturnal changes in pulmonary function following daytime exposure to aeroallergen. While increased TAA is consistent with bronchoconstriction, the changes may not be due solely to changes in $\mathrm{R}_{\mathrm{L}}$ and $\mathrm{C}_{\mathrm{dyn}}$. Regardless, the results provide compelling evidence that daytime exposure to aeroallergen in allergic NHP is associated with an increased incidence of nocturnal disturbances in breathing. Taken together, the results indicate that symptoms of nocturnal asthma can be induced in NHP, providing a model for elucidating the mechanisms underlying circadian manifestation of asthma symptoms.

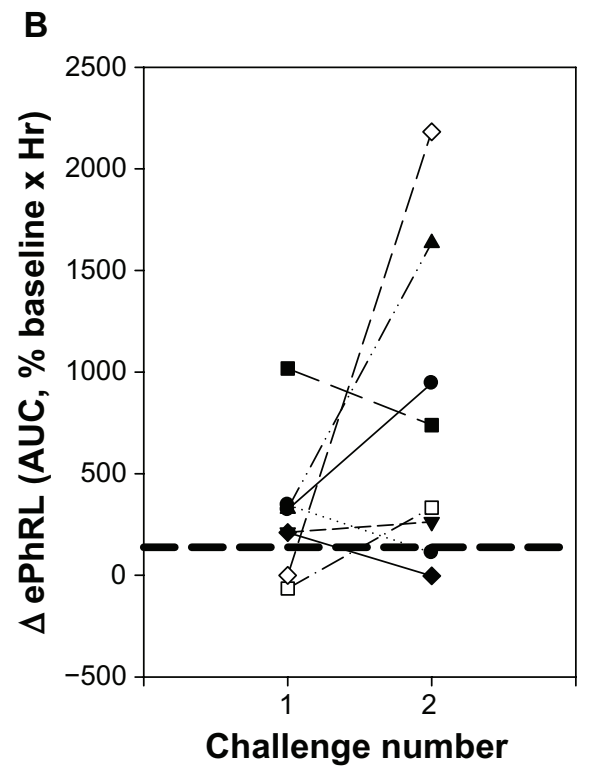

Figure 6 Reproducibility of the nocturnal response to HDM challenge. Eight animals were challenged with HDM on two different dates. AUC for (A) eBPRL and (B) ePhRL are shown. Symbols represent individual animals. Dash lines represent the upper limit of the $99 \%$ confidence intervals for the nonallergic group. The symbols above the dashed lines were classified as nocturnal asthmatic responses.

Abbreviations: eBPRL, baseline effort phase relation; ePhRL, effort phase relation; AUC, area under the curve; HDM, house dust mite. 


\section{Disclosure}

The authors report no conflicts of interest in this work.

\section{References}

1. National Health Interview Survey (NHIS) Data (2007 Data). 2007. Centers for Disease Control and Prevention.

2. Ballard RD, Saathoff MC, Patel DK, Kelly PL, Martin RJ. Effect of sleep on nocturnal bronchoconstriction and ventilatory patterns in asthmatics. J Appl Physiol. 1989;67(1):243-249.

3. Irvin CG, Pak J, Martin RJ. Airway-parenchyma uncoupling in nocturnal asthma. Am J Respir Crit Care Med. 2000;161(1):50-56.

4. Kelly EA, Houtman JJ, Jarjour NN. Inflammatory changes associated with circadian variation in pulmonary function in subjects with mild asthma. Clin Exp Allergy. 2004;34(2):227-233.

5. Kraft M, Vianna E, Martin RJ, Leung DY. Nocturnal asthma is associated with reduced glucocorticoid receptor binding affinity and decreased steroid responsiveness at night. JAllergy Clin Immunol. 1999; 103(1 Pt 1):66-71.

6. Smolensky MH, Lemmer B, Reinberg AE. Chronobiology and chronotherapy of allergic rhinitis and bronchial asthma. Adv Drug Deliv Rev. 2007;59(9-10):852-882.

7. Turner-Warwick M. Epidemiology of nocturnal asthma. Am J Med. 1988;85(1B):6-8.

8. Cochrane GM, Clark JH. A survey of asthma mortality in patients between ages 35 and 64 in the Greater London hospitals in 1971. Thorax. 1975;30(3):300-305.

9. Hetzel MR, Clark TJ, Branthwaite MA. Asthma: Analysis of sudden deaths and ventilatory arrests in hospital. $B r$ Med $J .1977 ; 1(6064)$ : 808-811.

10. Weitzman JB, Kanarek NF, Smialek JE. Medical examiner asthma death autopsies: A distinct subgroup of asthma deaths with implications for public health preventive strategies. Arch Pathol Lab Med. 1998;122(8):691-699.

11. Abraham WM, Watson H, Schneider A, King M, Yerger L, Sackner MA. Noninvasive ventilatory monitoring by respiratory inductive plethysmography in conscious sheep. J Appl Physiol. 1981;51(6):1657-1661.

12. Miller C, Hoffman AM, Hunter J. Thoracoabdominal asynchrony failed to grade airway obstructions in foals. J Appl Physiol. 2000;88(6): 2081-2087.

13. Pennock BE, Cox CP, Rogers RM, Cain WA, Wells JH. A noninvasive technique for measurement of changes in specific airway resistance. J Appl Physiol. 1979;46(2):399-406.

14. Millard RK. Inductive plethysmography components analysis and improved non-invasive postoperative apnoea monitoring. Physiol Meas. 1999;20(2):175-186.

15. Rusconi F, Gagliardi L, Aston H, Silverman M. Respiratory inductive plethysmography in the evaluation of lower airway obstruction during methacholine challenge in infants. Pediatr Pulmonol. 1995;20(6):396-402.

16. Tobin MJ, Jenouri G, Lind B, Watson H, Schneider A, Sackner MA. Validation of respiratory inductive plethysmography in patients with pulmonary disease. Chest. 1983;83(4):615-620.

17. Stromberg NO, Gustafsson PM. Breathing pattern variability during bronchial histamine and methacholine challenges in asthmatics. Respir Med. 1996;90(5):287-296.

18. Willis BC, Graham AS, Wetzel RL, Newth CJ. Respiratory inductance plethysmography used to diagnose bilateral diaphragmatic paralysis: A case report. Pediatr Crit Care Med. 2004;5(4):399-402.

19. Black J, Baxter-Jones AD, Gordon J, Findlay AL, Helms PJ. Assessment of airway function in young children with asthma: Comparison of spirometry, interrupter technique, and tidal flow by inductance plethysmography. Pediatr Pulmonol. 2004;37(6):548-553.

20. Brown KA, Aoude AA, Galiana HL, Kearney RE. Automated respiratory inductive plethysmography to evaluate breathing in infants at risk for postoperative apnea. Can J Anaesth. 2008;55(11):739-747.
21. Cantineau JP, Escourrou P, Sartene R, Gaultier C, Goldman M. Accuracy of respiratory inductive plethysmography during wakefulness and sleep in patients with obstructive sleep apnea. Chest. 1992;102(4): $1145-1151$.

22. Hammer J, Newth CJ. Assessment of thoraco-abdominal asynchrony. Paediatr Respir Rev. 2009;10(2):75-80.

23. Kaplan V, Zhang JN, Russi EW, Bloch KE. Detection of inspiratory flow limitation during sleep by computer assisted respiratory inductive plethysmography. Eur Respir J. 2000;15(3):570-578.

24. Kohyama J, Shiiki T, Shimohira M, Hasegawa T. Asynchronous breathing during sleep. Arch Dis Child. 2001;84(2):174-177.

25. Springer C, Godfrey S, Vilozni D, Bar-Yishay E, Noviski N, Avital A. Comparison of respiratory inductance plethysmography with thoracoabdominal compression in bronchial challenges in infants and young children. Am J Respir Crit Care Med. 1996;154(3 Pt 1): 665-669.

26. Whyte KF, Gugger M, Gould GA, Molloy J, Wraith PK, Douglas NJ. Accuracy of respiratory inductive plethysmograph in measuring tidal volume during sleep. J Appl Physiol. 1991;71(5):1866-1871.

27. Sackner MA, Gonzalez H, Rodriguez M, Belsito A, Sackner DR, Grenvik S. Assessment of asynchronous and paradoxic motion between rib cage and abdomen in normal subjects and in patients with chronic obstructive pulmonary disease. Am Rev Respir Dis. 1984;130(4):588-593.

28. Hillman DR, Prentice L, Finucane KE. The pattern of breathing in acute severe asthma. Am Rev Respir Dis. 1986;133(4):587-592.

29. Ringel ER, Loring SH, McFadden ER Jr, Ingram RH Jr. Chest wall configurational changes before and during acute obstructive episodes in asthma. Am Rev Respir Dis. 1983;128(4):607-610.

30. Litman RS, Kottra JA, Gallagher PR, Ward DS. Diagnosis of anestheticinduced upper airway obstruction in children using respiratory inductance plethysmography. J Clin Monit Comput. 2002;17(5): 279-285.

31. De GA, Groswasser J, Bersini H, Mathys P, Kahn A. Detection of obstructive apnea events in sleeping infants from thoracoabdominal movements. J Sleep Res. 2002;11(2):161-168.

32. Coffman RL, Hessel EM. Nonhuman primate models of asthma. $J$ Exp Med. 2005;201(12):1875-1879.

33. van Scott MR, Hooker JL, Ehrmann D, et al. Dust mite-induced asthma in Cynomolgus monkeys. J Appl Physiol. 2004;96(4):1433-1444.

34. van Scott MR, Aycock D, Cozzi E, Salleng K, Stallings HW III. Separation of bronchoconstriction from increased ventilatory drive in a nonhuman primate model of chronic allergic asthma. J Appl Physiol. 2005;99(6):2080-2086.

35. Ayanoglu G, Desai B, Fick RB, et al. Longitudinal characterization of cellular and molecular markers in a primate model of chronic house dust mite asthma. (Submitted) 2010.

36. Erpenbeck VJ, Schmidt R, Gunther A, Krug N, Hohlfeld JM. Surfactant protein levels in bronchoalveolar lavage after segmental allergen challenge in patients with asthma. Allergy. 2006;61(5):598-604.

37. Joad JP, Kott KS, Bric JM, et al. Structural and functional localization of airway effects from episodic exposure of infant monkeys to allergen and/or ozone. Toxicol Appl Pharmacol. 2006;214(3):237-243.

38. Kott KS, Pinkerton KE, Bric JM, Plopper CG, Avadhanam KP, Joad JP. Methacholine responsiveness of proximal and distal airways of monkeys and rats using videomicrometry. J Appl Physiol. 2002; 92(3):989-996.

39. Keenan DB, Wilhelm FH. Adaptive and wavelet filtering methods for improving accuracy of respiratory measurement. Biomed Sci Instrum. 2005; 41:37-42.

40. Keenan DB, Wilhelm FH. Classification of locomotor activity by acceleration measurement: Validation in Parkinson disease. Biomed Sci Instrum. 2005;41:329-334.

41. Prisk GK, Hammer J, Newth CJ. Techniques for measurement of thoracoabdominal asynchrony. Pediatr Pulmonol. 2002;34(6):462-472.

42. Sackner MA, Watson H, Belsito AS, et al. Calibration of respiratory inductive plethysmograph during natural breathing. $J$ Appl Physiol. 1989;66(1):410-420. 
43. Sartene R, Dartus C, Bernard JL, Mathieu M, Goldman MD. Comparison of thoracoabdominal calibration methods in normal human subjects. J Appl Physiol. 1993;75(5):2142-2150.

44. Allen JL, Wolfson MR, McDowell K, Shaffer TH. Thoracoabdominal asynchrony in infants with airflow obstruction. Am Rev Respir Dis. 1990;141(2):337-342.

45. Warren RH, Fewell JE, Alderson SH. Calibration of respiratory inductive plethysmography during quiet and active sleep in lambs. $J$ Dev Physiol. 1989;12(6):347-352.

46. Stromberg NO, Gustafsson PM. Hyperventilation during bronchial challenges in asthmatics: Reproducibility and assessment of contributing factors. Respir Med. 1996;90(5):297-306.

47. Fujimori K, Satoh M, Arakawa M. Ventilatory response to continuous incremental changes in respiratory resistance in patients with mild asthma. Chest. 1996;109(6):1525-1531.

48. Storms WW, Bodman SF, Nathan RA, Byer P. Nocturnal asthma symptoms may be more prevalent than we think. JAsthma. 1994;31(4): 313-318.
49. Shigemitsu H, Afshar K. Nocturnal asthma. Curr Opin Pulm Med. 2007;13(1):49-55.

50. Fix A, Sexton M, Langenberg P, Santanello N, Hyndman S, Williams R. The association of nocturnal asthma with asthma severity. J Asthma. 1997;34(4):329-336.

51. Robertson DG, Kerigan AT, Hargreave FE, Chalmers R, Dolovich J. Late asthmatic responses induced by ragweed pollen allergen. J Allergy Clin Immunol. 1974;54(4):244-254.

52. O'Byrne PM, Dolovich J, Hargreave FE. Late asthmatic responses. Am Rev Respir Dis. 1987;136(3):740-751.

53. Nabe T, Zindl CL, Jung YW, et al. Induction of a late asthmatic response associated with airway inflammation in mice. Eur J Pharmacol. 2005; 521(1-3):144-155.

\section{Publish your work in this journal}

The Journal of Asthma and Allergy is an international, peer-reviewed open-access journal publishing original research, reports, editorials and commentaries on the following topics: Asthma; Pulmonary physiology; Asthma related clinical health; Clinical immunology and the immunological basis of disease; Pharmacological interventions and new therapies. Issues of patient safety and quality of care will also be considered. The manuscript management system is completely online and includes a very quick and fair peer-review system, which is all easy to use. Visit http://www.dovepress.com/testimonials.php to read real quotes from published authors.

Submit your manuscript here: http://www.dovepress.com/journal-of-asthma-and-allergy-journal 